\title{
Two-Tier Tissue Decomposition for Histopathological Image Representation and Classification
}

\author{
Tunc Gultekin, Can Fahrettin Koyuncu, Cenk Sokmensuer, and Cigdem Gunduz-Demir*, Member, IEEE
}

\begin{abstract}
In digital pathology, devising effective image representations is crucial to design robust automated diagnosis systems. To this end, many studies have proposed to develop object-based representations, instead of directly using image pixels, since a histopathological image may contain a considerable amount of noise typically at the pixel-level. These previous studies mostly employ color information to define their objects, which approximately represent histological tissue components in an image, and then use the spatial distribution of these objects for image representation and classification. Thus, object definition has a direct effect on the way of representing the image, which in turn affects classification accuracies. In this paper, our aim is to design a classification system for histopathological images. Towards this end, we present a new model for effective representation of these images that will be used by the classification system. The contributions of this model are twofold. First, it introduces a new two-tier tissue decomposition method for defining a set of multityped objects in an image. Different than the previous studies, these objects are defined combining texture, shape, and size information and they may correspond to individual histological tissue components as well as local tissue subregions of different characteristics. As its second contribution, it defines a new metric, which we call dominant blob scale, to characterize the shape and size of an object with a single scalar value. Our experiments on colon tissue images reveal that this new object definition and characterization provides distinguishing representation of normal and cancerous histopathological images, which is effective to obtain more accurate classification results compared to its counterparts.
\end{abstract}

Index Terms-Automated cancer diagnosis, blob, digital pathology, histopathological image representation, tissue decomposition model.

\section{INTRODUCTION}

D IFFERENT tissues come together to form an organ in the body. Depending on its type, cancer causes different kinds of changes in these tissues. Thus, in cancer diagnosis and grading, pathologists examine the tissue changes considering the tissue type and may attach different levels of importance to the changes occurred in different tissue regions. For example,

Manuscript received August 01, 2014; accepted August 25, 2014. Date of publication September 04, 2014; date of current version December 24, 2014. Asterisk indicates corresponding author.

T. Gultekin and C. F. Koyuncu are with the Department of Computer Engineering, Bilkent University, TR-06800 Ankara, Turkey (e-mail: tunc.gultekin@bilkent.edu.tr; koyuncu@cs.bilkent.edu.tr).

C. Sokmensuer is with the Department of Pathology, Hacettepe University Medical School, TR-06100 Ankara, Turkey (e-mail: csokmens@hacettepe.edu. tr).

${ }^{*}$ C. Gunduz-Demir is with the Department of Computer Engineering, Bilkent University, TR-06800 Ankara, Turkey (e-mail: gunduz@cs.bilkent.edu.tr).

Color versions of one or more of the figures in this paper are available online at http://ieeexplore.ieee.org.

Digital Object Identifier 10.1109/TMI.2014.2354373

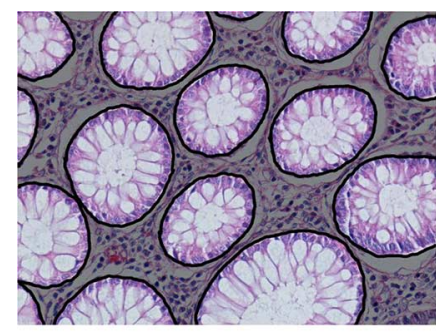

(a)

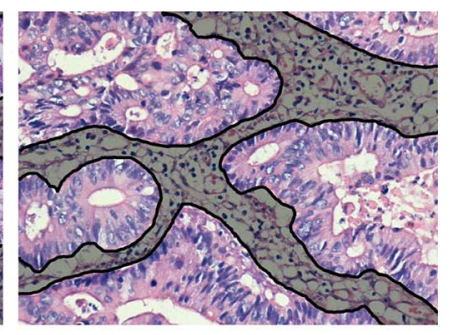

(b)
Fig. 1. (a) A normal and (b) a cancerous colon tissue image. On these images, epithelial (nonshaded) and connective (gray-shaded) tissue regions are shown.

colon contains epithelial and connective tissues. In the diagnosis of colon adenocarcinoma, which accounts for $90 \%-95 \%$ of all colorectal cancers, examining epithelial tissue regions is more important since this cancer type originates from the epithelial tissue and causes substantial changes in these regions (Fig. 1).

In the digital pathology literature, classification studies extract mathematical features to model the tissue changes and use them to classify histopathological images. The previous studies have used two main approaches for feature extraction. In the first approach, they extract features for each image pixel using various methods including intensity histograms [1], [2], co-occurrence matrices [3], [4], filters [5], [6], and local binary patterns [7], [8]. They then define global features accumulating the pixels' features over an entire image. However, the image may contain local regions corresponding to different tissue types, which may show different image characteristics. Thus, globally accumulating the pixels' features without considering the local regions may weaken the representative power of these features and may lead to misclassifications.

In the second approach, the previous studies define objects in an image to represent histological tissue components and work on these objects instead of image pixels. The majority of these studies define objects for nucleus components and characterize the image with global features extracted from a graph of these components [9]-[11]. In our recent studies, we define multityped objects, for also representing stromal and luminal components, and quantify their spatial distributions using graphs [12], [13] as well as defining object textures [14]. Similarly, all of these studies define objects over an entire image and characterize the image without making any distinction between the objects defined in the local regions of different characteristics.

In this paper, our aim is to design a classification system for histopathological images. To this end, we propose a new two- 
tier tissue decomposition method for histopathological image representation, which will be used by the classification system. In the first tier of this method, we decompose an image into a set of local regions (objects) that show similar texture characteristics. Then, in its second tier, we put these objects into further categories based on their shape and size properties that we quantify by introducing a new metric. Finally, we construct a graph on the objects and use its edge distribution for image representation.

The main contributions of this work are twofold. First, it proposes a new tissue decomposition method for object definition. Different than the previous studies that defined nucleus locations as their objects, it identifies multityped objects and uses their distribution for tissue quantification. The proposed model is also different than our previous studies that used multityped objects, in the sense of defining the objects. Our previous studies [12]-[14] defined their objects by locating circles of various radii on the dominant colors (white, pink, and purple) of histopathological images stained with hematoxylin-and-eosin. Since different tissues (e.g., epithelial and connective tissues shown in Fig. 1) show similar color distributions, there is no distinction between the types of the circular objects defined on the local regions of different tissue types. On the other hand, the decomposition method proposed by this current work uses texture to identify objects in its first tier. Since texture is more distinctive than color for these local regions, the objects are expected to show more variety among the local regions of different tissue types. This helps better represent histopathological images and more accurately classify them. Additionally, our previous studies restrict objects to have regular shapes (simply circles). On the other hand, our new decomposition method does not have such kind of restriction and allows us to define irregular-shaped objects that approximately represent histological tissue components (e.g., cell nuclei) and local tissue subregions of different characteristics (e.g., epithelial cell regions). Since the second tier of the proposed method further categorizes these irregular-shaped objects based on their shape and size, it is expected to define more distinguishing objects for histopathological image representation.

As its second contribution, this work introduces a new metric, which we call "dominant blob scale," to quantify the shape and size of the irregular-shaped objects, each of which may approximately represent a histological tissue component or a local tissue subregion. To this end, it defines a set of ring-like filters with different sizes, iteratively convolves each object with these filters, and quantifies the object with the size of the filter that covers this object. This metric uses the idea of blob definition, which is frequently employed in different computer vision applications such as salient point localization [15]-[17] and object tracking [18], [19]. Blobs have also been used for feature extraction; previous studies [20]-[22] use blobs to define closed areas from which features will be extracted. On the other hand, different than these previous studies, our current work directly uses blobs (their scales) in an iterative algorithm to define a feature that quantifies the size and shape of an irregular-shaped object with a single scalar value.

Working on 3236 microscopic colon tissue images, our experiments demonstrate that the distribution of the multityped

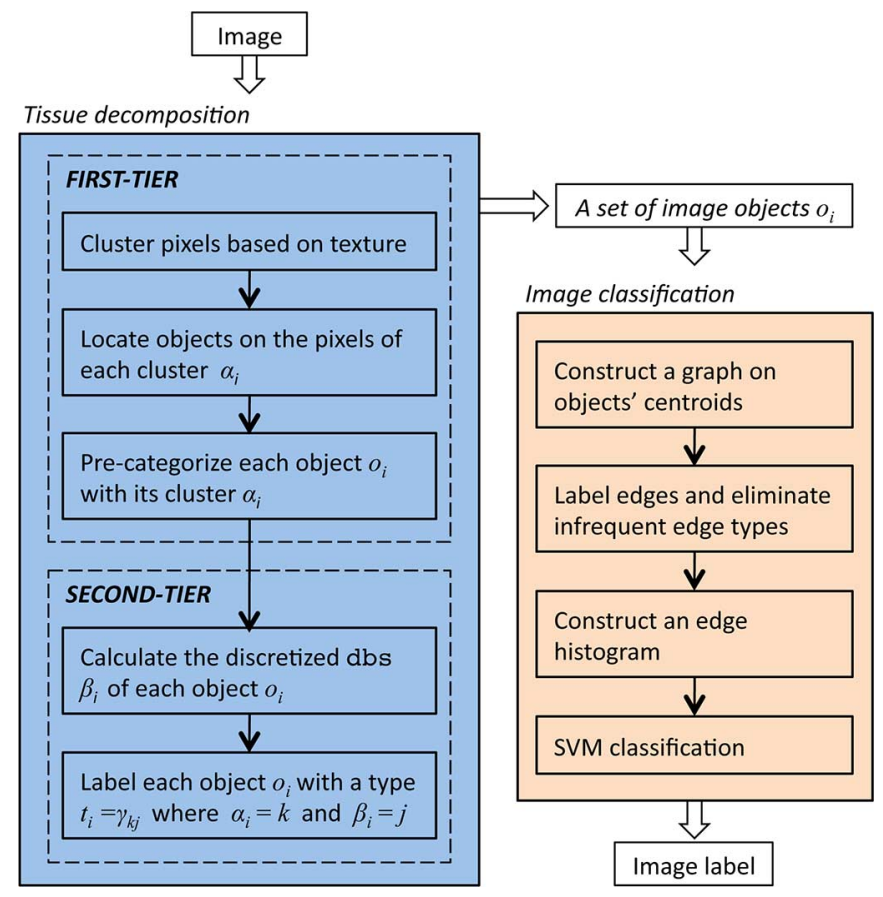

Fig. 2. Schematic overview of the proposed approach.

objects, defined and categorized by our new decomposition method, is more effective in histopathological image representation and gives more accurate results in classification compared to its counterparts.

\section{Methodology}

In this paper, our aim is to design an effective model for the purpose of histopathological image classification. To this end, we propose a new representation that decomposes an image into multityped objects and use the spatial distribution of these objects in classification. In this representation, the ideal way was to define an object type for each of the histological component and tissue subregion types as well as to exactly localize each of these components and subregions. However, this exact representation would arise a very difficult segmentation problem, even for a human eye. Thus, we devise an approximate representation, in which there may exist one-to-one, one-to-many, and many-to-one correspondences between the object types and the types of the components/subregions. Moreover, in our representation, the components/subregions are only approximately localized. It is worth to noting that the defined objects may not be directly used by pathologists, but the classification system using this object representation may assist them in cancer diagnosis and grading.

The proposed model has two main steps (Fig. 2): tissue decomposition and image classification. For the first step, we devise a new two-tier method. In the first tier of this method, we locate objects on an image and precategorize them based on their texture characteristics. Then, in its second tier, we further categorize the objects based on their shapes and sizes, which we quantify by introducing the dominant blob scale metric. In the second step, we construct a graph on the identified objects' centroids, label graph edges according to the types of their end 


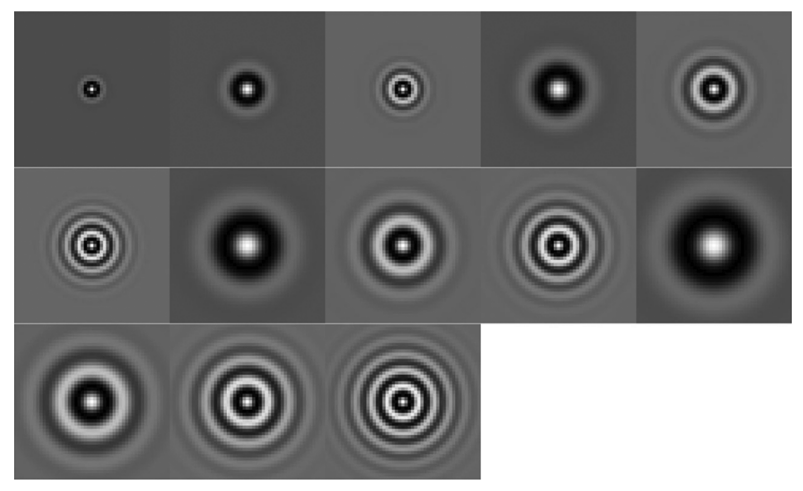

Fig. 3. Illustration of the 13 rotational invariant filters defined in the Schmid's filter bank [23].

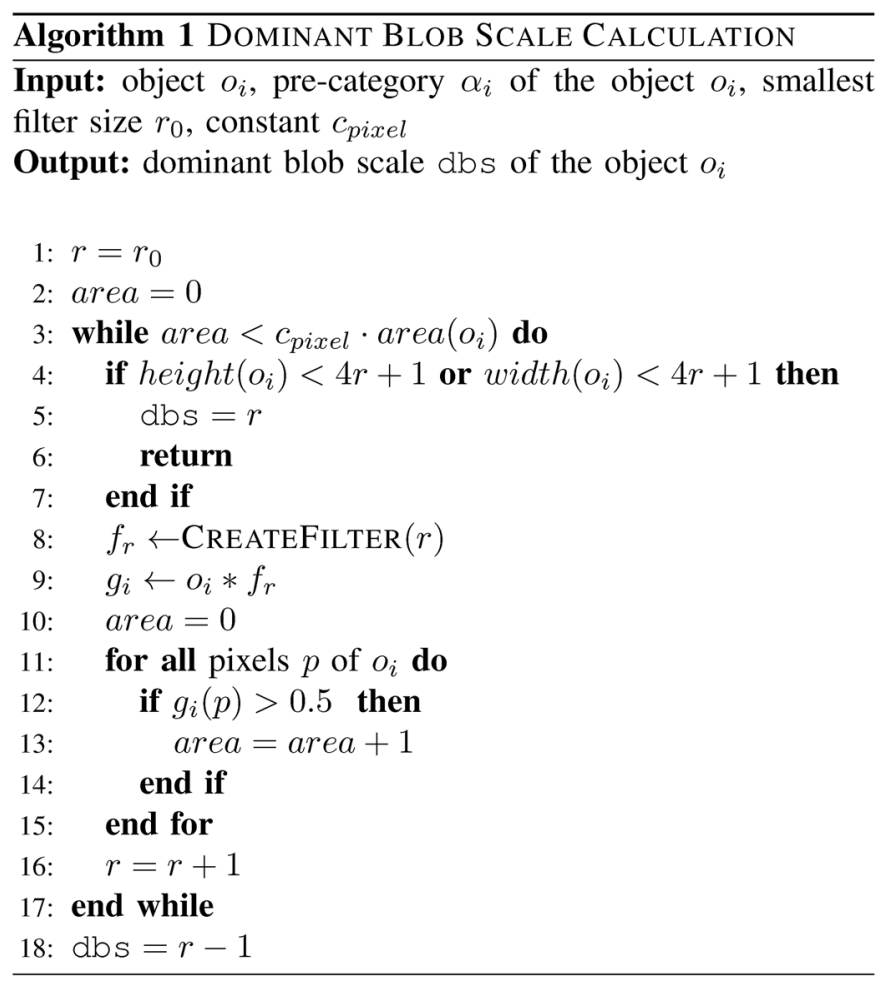

nodes, and extract a feature set from the histogram of the edge types. We then use this feature set to classify tissue images. These two main steps are further detailed in the following subsections.

\section{A. Tissue Decomposition}

We decompose an image $I$ into the characterized objects using a two-tier method. In the first tier, we make use of texture characteristics. To this end, we convolve the normalized gray intensities of image pixels with the 13 rotational invariant filters (Fig. 3) defined in the Schmid's filter bank [23]. We then assign each pixel to one of the $K$ clusters, learned by the k-means algorithm ${ }^{1}$, according to the pixel's filter outputs.

\footnotetext{
${ }^{1}$ In our experiments, we randomly selected 20 training images from each class and learned clustering vectors by running the k-means algorithm on the pixels of these training images.
}

Thus, each cluster represents a set of pixels showing similar texture properties.

Subsequently, we find connected components on the pixels of each cluster ${ }^{2}$ and take the components whose areas are greater than an area threshold $T_{\text {area }}$ as image objects. At the end of the first tier, we identify a set of image objects $\mathcal{O}(I)=\left\{o_{i}\right\}$, each of which is characterized with its cluster (precategory) $\alpha_{i} \in\{1, \ldots, K\}$. Note that these objects may correspond to individual histological tissue components or local subregions of different tissue characteristics. For instance, the first row of Fig. 4 shows a normal, a low-grade cancerous, and a high-grade cancerous tissue image and its second row visualizes the objects found in the first tier (objects of each precategory $\alpha_{i}$ are indicated with a different color). As shown in these images, red objects mostly represent individual stromal cell nuclei whereas blue objects mostly correspond to epithelial cell nucleus regions.

In the second tier, for each object $o_{i}$, we compute the dominant blob scale metric (dbs), whose calculation details will be given in the next subsection. We propose this metric to quantify the object's shape and size with a single value. In this work, we use the dbs metric to group objects belonging to the same precategory $\alpha_{i}$ into subcategories. For this purpose, we quantize the $\mathrm{dbs}$ of the objects located on training images by the k-means algorithm and learn three clustering vectors corresponding to small, medium, and large scaled objects. Then, we compute the discretized dbs of an object, $\beta_{i} \in$ \{small, medium, large $\}$, by assigning this object into one of these clusters. The last row of Fig. 4 shows subcategories $\beta_{i}$ for the objects belonging to the yellow precategory $\alpha_{i}$ of the second row of this figure. Note that in this paper, we employ a relatively simple method to define the subcategories using the dbs metric. However, it is also possible to design different methods using this metric; its uses could be considered as a possible future work.

After identifying the subcategories, we label each object with respect to its precategory $\alpha_{i}$ and its discretized dbs $\beta_{i}$. In particular, we label the object $o_{i}$ with a type $t_{i}=\gamma_{k j}$ if $\alpha_{i}=k$ and $\beta_{i}=j$. Thus, each object is labeled with one of the $3 K$ different types.

1) Dominant Blob Scale: To calculate the dbs of an object $o_{i}$, we iteratively convolve its binary representation with a set of ring-like filters of different sizes. In this binary representation, pixels belonging to $o_{i}$ are marked as 1 and all others as 0 . Then, we define $\operatorname{dbs}\left(o_{i}\right)$ as the size of the filter that first covers the object $o_{i}$ according to Definition 1 .

Definition 1: An object $o_{i}$ is said to be covered by a filter $f_{r}$ with respect to a constant $c_{\text {pixel }}$ if and only if $c_{\text {pixel }}$ percent of its pixels are covered by $f_{r}$.

Definition 2: A pixel $p$ is said to be covered by a filter $f_{r}$ if and only if the filter output for $p$ is greater than 0.5 .

The pseudocode for the dominant blob scale calculation is given in Algorithm 1. This algorithm takes four inputs. The first two are the object $o_{i}$ and its precategory $\alpha_{i}$. The next one is the minimum size $r_{0}$ of the filter from which iterations start. The last parameter is the constant $c_{\text {pixel }}$ used in Definition 1. Then, the

\footnotetext{
${ }^{2}$ For a cluster, connected component labeling scans all pixels of that cluster and puts the pixels connected to each other into a single component.
} 


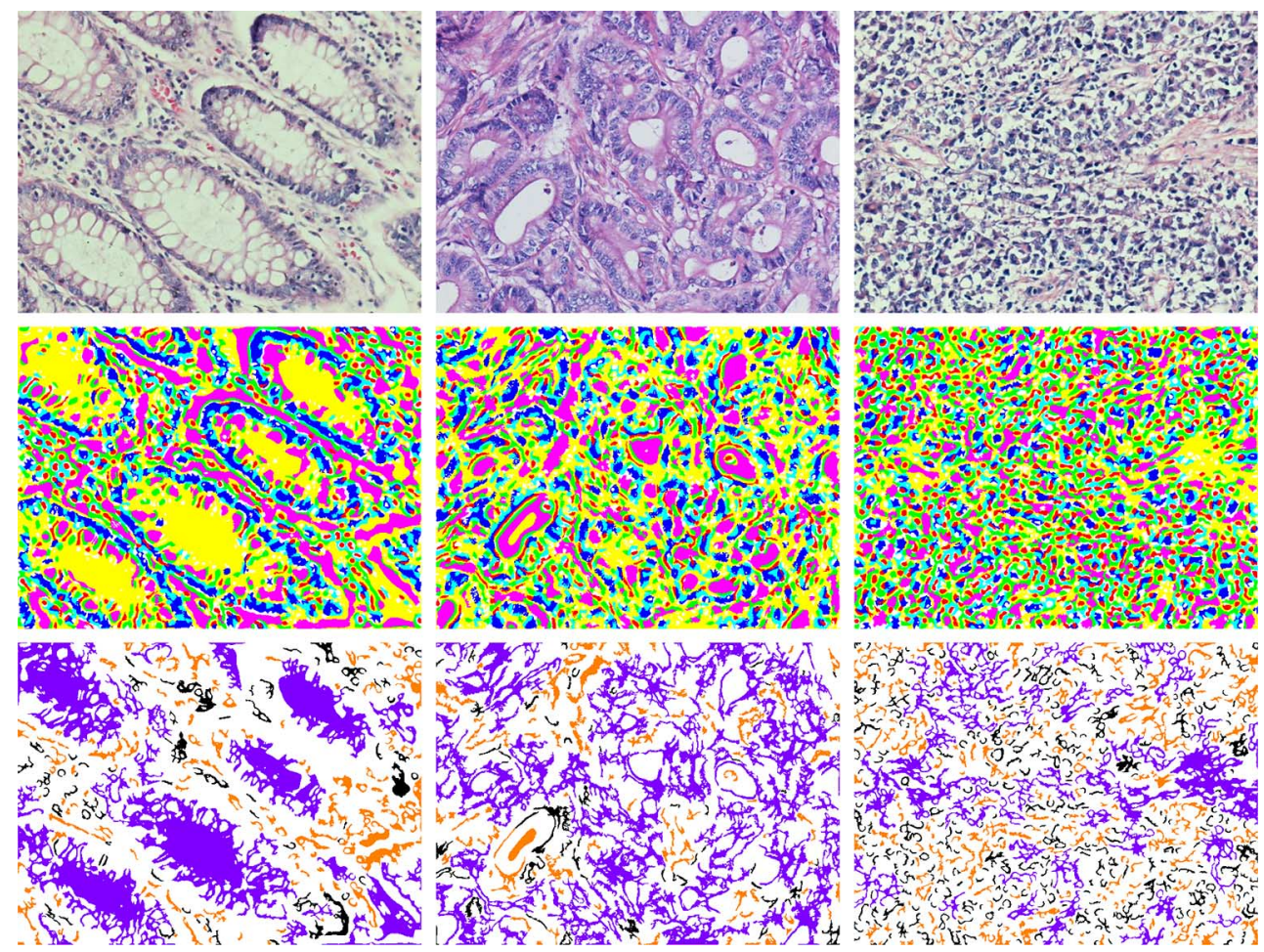

Fig. 4. First row (from left to right): normal, low-grade cancerous, and high-grade cancerous tissue images. Second row: objects found in the first tier, with their precategories indicated. Note that the objects smaller than an area threshold $T_{\text {area }}$ are eliminated from the object set. Such objects are not shown in these images. Third row: subcategories that the dbs metric defines for the objects belonging to the yellow precategory of the second row. In the third row, orange, black, and purple indicate small, medium, and large objects, respectively.
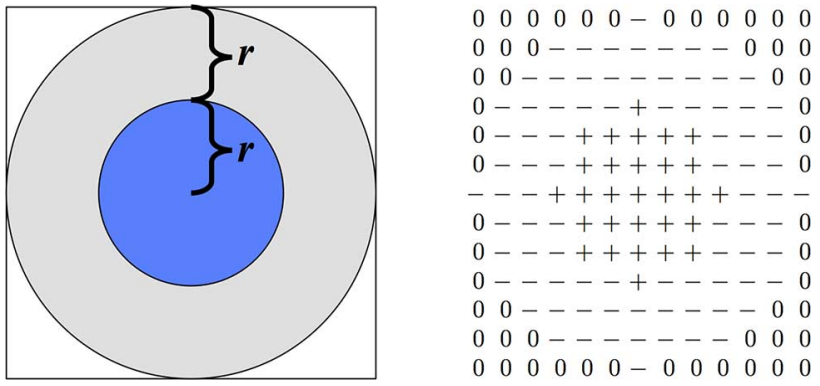

Fig. 5. Ring-like filter $f_{r}$ defined for size $r$.

algorithm outputs the dominant blob scale of the input object, $\mathrm{dbs}\left(o_{i}\right)$, which is used to calculate the object's discretized dbs $\beta_{i}$ and hence its type $t_{i}$.

2) Ring-Like Filters: We create a filter $f_{r}$ with a size of $r$ as follows. The filter includes a positive disk with a radius of $r$ at its center and a negative ring with a width of $r$ surrounding this disk (see Fig. 5). In this filter, we assign a positive value $u_{p}$ to every entry in the disk such that their sum will be 1. Likewise, we assign a negative value $u_{n}$ to every entry in the ring such that their sum will be -1 .

The iterative dbs calculation algorithm, which uses these filters, starts covering object's pixels from its corners thanks to the existence of a positive disk surrounded by a negative ring. That is, a pixel close to a corner is covered earlier than a pixel far from corners since the filter output of a covered pixel is greater than 0.5 , which indicates that a substantial part of the negative ring should be convolved with nonobject pixels (see the first row

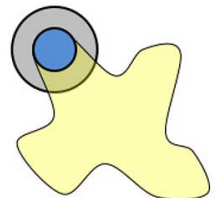

covered
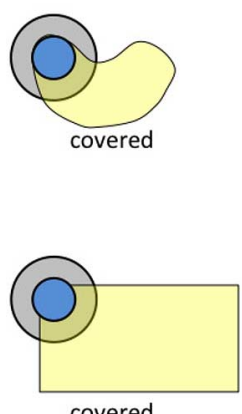

covered
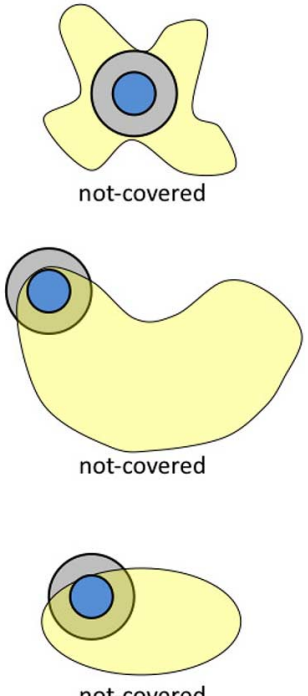

not-covered
Fig. 6. First row: pixels close to a corner are covered earlier than those far from corners. Second row: pixels of smaller objects are covered earlier than those of larger ones. Third row: pixels of more rectangular-like objects are covered earlier than those of rounder ones.

of Fig. 6). Due to the same reason, pixels of smaller objects are covered earlier than those of larger ones (see the second row of Fig. 6) and pixels of more rectangular-like objects are covered earlier than those of rounder ones (see the last row of Fig. 6).

This results in the iterative algorithm to yield larger dbs values for larger and rounder objects compared to smaller and 

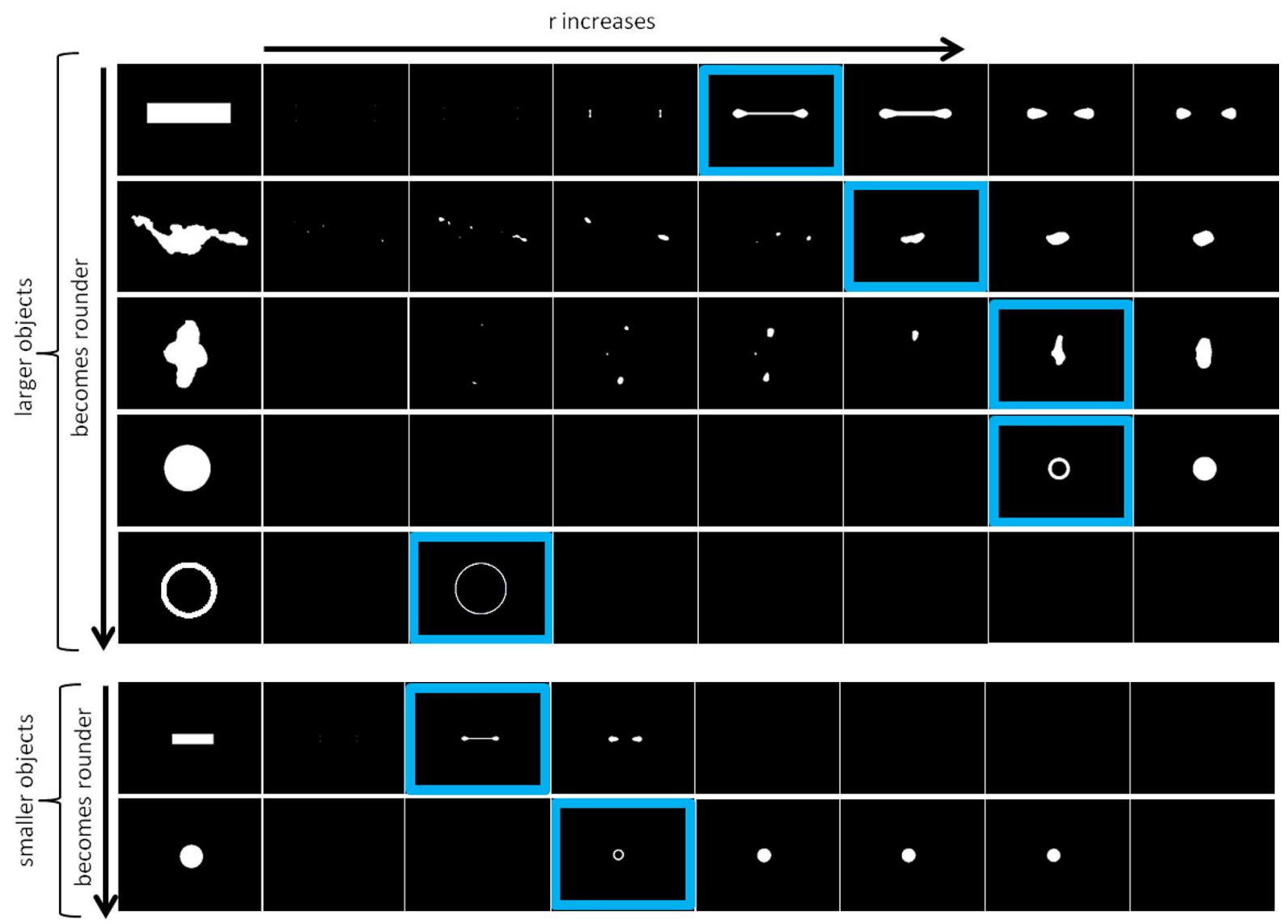

Fig. 7. Illustration of dbs calculation for objects of different sizes and shapes. The outputs obtained by the filter of size $r$ that is used to define the dbs of these objects are indicated with blue boundaries. As shown in this figure, rounder objects are covered by relatively large filters, leading to larger dbs values. Similarly, larger objects are covered by larger filters compared to smaller ones.

more rectangular-like ones. Remember that an object is covered by a filter, whose size will determine the dbs of this object, when $c_{\text {pixel }}$ percent of its pixels are covered by this filter. Therefore, the ratio of the covered pixels, and hence the dbs value, gives information about the object's size and shape. For objects of different sizes and shapes, Fig. 7 illustrates the responses of different-sized filters as well as indicates the one whose size $r$ is used to define the dbs of these objects with blue boundaries. The objects shown in the first four rows of this figure have exactly the same size but their shapes become rounder from top to bottom. These objects are larger than those given in the last two rows of this figure. As shown in Fig. 7, rounder objects are covered by relatively large filters, leading to larger dbs values. Similarly, larger objects are covered by larger filters compared to smaller ones.

\section{B. Image Representation and Classification}

We characterize an image $I$ by making use of the spatial distribution of its objects $\mathcal{O}(I)=\left\{o_{i}\right\}$. To this end, we construct a graph $G=(V, E)$ on the objects' centroids by Delaunay triangulation. In this graph, the vertex set $V$ contains every object $o_{i} \in \mathcal{O}(I)$ and the edge set includes the triangle edges $e\left(o_{u}, o_{v}\right)$ that are labeled with respect to their end nodes. Particularly, we label the edge $e\left(o_{u}, o_{v}\right)$ with a type $\tau\left(t_{u}, t_{v}\right)$, where $t_{u}$ and $t_{v}$ denote the types of the objects $o_{u}$ and $o_{v}$. Then, we use the histogram of the edge types to represent the image $I$. Thus, this
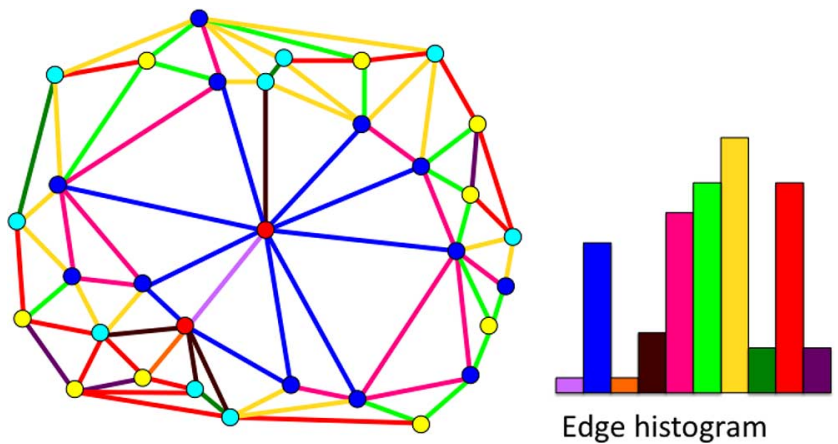

Edge histogram

Fig. 8. Illustration of Delaunay graph construction and feature extraction. In this figure, object centroids are shown with circles and only four object types are used for the sake of simplicity. Object types and edge types are illustrated with different colors.

representation encodes the neighborhood information between the adjacent objects. Fig. 8 illustrates the construction of a Delaunay graph on example objects. For the sake of simplicity, it shows objects as circles and uses only four object types. This figure also visualizes histogram creation from the edge types.

This representation has $3 K \cdot(3 K+1) / 2$ features since there exist $3 K$ different object types; here $K$ is the number of precategories (clusters) found in the first tier of tissue decomposition. This dimension is usually high and may lead to curse-of-dimensionality. Thus, we implement a method that automatically re- 
duces the dimension on the training images. In this method, we eliminate an edge type $\tau$ from representation if the number of edges of this type $\tau$ is smaller than an edge threshold $T_{\text {edge }}$ for every image in the training set. Then, we classify an image by a support vector machine that uses the histogram of the uneliminated edge types as its features. This classifier uses a linear kernel, $k(x, y)=x^{T} y+c$, which is commonly used by support vector machines.

\section{EXPERIMENTS}

\section{A. Dataset}

We test our method on colon tissue images acquired using a Nikon Coolscope Digital Microscope with a $20 \times$ objective lens and at $640 \times 480$ pixel resolution. These tissues were stained by the routinely used hematoxylin-and-eosin technique and labeled with one of the three classes, which are normal, low-grade cancerous, and high-grade cancerous. This dataset includes 3236 images taken from 258 patients. We randomly divide these patients into two groups and use images of the first group in the training set and images of the other in the test set. The training set includes 510 normal, 859 low-grade cancerous, and 275 high-grade cancerous tissue images. The test set includes 491 normal, 844 low-grade cancerous, and 257 high-grade cancerous tissue images.

In our experiments, we use the accuracy (the ratio of the correctly classified samples) to measure the classification performance. Here it is worth to noting that there is no ground truth for object segmentation and labeling since their exact localization is quite challenging even for a human eye. Indeed, this is also our main motivation behind defining the objects, which approximately represent histological tissue components and local tissue subregions. The proposed object representation is used to define features for classification. Thus, the classification performance might be considered as an indicator for the effectiveness of this representation, and hence, for the effectiveness of object segmentation and labeling.

\section{B. Comparisons}

We compare our proposed two-tier method with two groups of algorithms. In the first group, we divide an image into grids ${ }^{3}$ and quantify each grid by extracting pixel-based features. Then, we represent the image with the average of these grid features. In our experiments, we consider four types of features extracted using intensity histograms, co-occurrence matrices, Gabor filters, and local binary patterns. The details of these algorithms can be found in [14].

The second group includes object-based algorithms. In the first algorithm, we use features extracted from a Delaunay triangulation constructed on only nucleus tissue objects (singletyped objects). These features include the average degree, average clustering coefficient, and diameter of the Delaunay graph as well as the average, standard deviation, minimum-to-maximum ratio, and disorder of edge lengths and triangle areas. The other algorithms are those that we previously implemented in

\footnotetext{
${ }^{3}$ For each method in this group, the grid size is selected using threefold crossvalidation. The considered values are $\{10,20,40,80\}$.
}

our research group. In these algorithms, we also defined multityped objects and used their distribution for image representation. However, we used a single-step object definition procedure, in which image pixels are clustered based on their colors, instead of their textures, and circles are located on each of these clusters. Each object is then characterized with the cluster on which it is located. Thus, each object has a regular shape and typically corresponds to an entire or a partial histological tissue component but does not usually represent a local tissue subregion. Moreover, in these previous studies, we defined a single type for each of the cluster without considering the objects' scales. Particularly, in the ColorGraph algorithm, we extract global features from a Delaunay triangulation constructed on circular objects of three different types, which are defined for the three dominant colors (purple, pink, and white) in a hematoxylin-and-eosin stained histopathological image [12]. In the HybridModel algorithm, we search a query subgraph of such circular objects on the entire graph of an image and use the graph edit distance metric for image quantification [13]. In the LocalObjectPattern algorithm, we define a texture on the circular objects with the aforementioned three types and use this texture metric for quantification [14].

\section{Parameter Selection}

The proposed two-tier method has four external model parameters: cluster number $K$, area threshold $T_{\text {area }}$, covered pixel percentage $c_{\text {pixel }}$, and edge threshold $T_{\text {edge }}$. Besides, the support vector machine classifier has an additional parameter $C$. In our experiments, we select these parameters applying threefold cross-validation on the training images; this selection does not use any test images at all. In particular, we consider different values of these parameters and select the combination for which threefold cross-validation yields the highest classbased average accuracy. The sets of the parameter values that we consider are $K=\{2,4,6,8\}, T_{\text {area }}=\{20,30,40,50\}$, $c_{\text {pixel }}=\{1,5,10,25\}, T_{\text {edge }}=\{10,15,20,25\}$, and $C=$ $\{0.005,0.01,0.05,0.1,0.5,1,2, \ldots, 10,20, \ldots, 100\}$. The selected values are $K=6, T_{\text {area }}=40, c_{\text {pixel }}=5, T_{\text {edge }}=20$, and $C=0.01$. We also use threefold cross validation to select the parameter values for the comparison algorithms.

\section{RESULTS}

In Table I, we report the test set accuracies obtained by our proposed two-tier tissue decomposition model and the comparison algorithms. This table shows that the proposed model leads to high accuracies $(>93 \%)$ for all of the classes. This table also shows that misclassifications occur mostly for low-grade and high-grade cancerous tissues. This is indeed consistent with the current practice, in which grading is much more challenging compared to diagnosis. The reason might be that normal tissues have distinctive appearances whereas the appearances of cancerous tissues may vary related to cancer characteristics. This might also be the main reason for our model to define more distinguishing objects for normal tissues, which help obtain better performance for their classification.

The proposed model yields the highest overall accuracy compared to the other algorithms. The pixel-based comparison algorithms represent an image by accumulating features defined at 
TABLE I

Test Set Results of the Proposed Two-Tier Tissue Decomposition MODEL AND THE COMPARISON ALGORITHMS

\begin{tabular}{|l|c|c|c|c|}
\hline & Normal & Low & High & Overall \\
\hline Two-tier model & 99.18 & 93.83 & 93.77 & 95.47 \\
\hline Pixel-based approaches & & & & \\
\hline IntensityHistogram & 78.82 & 74.17 & 78.60 & 76.32 \\
\hline CooccurrenceMatrix & 87.58 & 84.12 & 85.60 & 85.43 \\
\hline GaborFilter & 91.24 & 82.23 & 78.60 & 84.42 \\
\hline LocalBinaryPattern & 92.67 & 73.46 & 80.54 & 80.53 \\
\hline Object-based approaches & & & & \\
\hline DelaunayTriangulation & 89.61 & 71.56 & 87.55 & 79.71 \\
\hline ColorGraph [12] & 92.67 & 82.46 & 86.38 & 86.24 \\
\hline HybridModel [13] & 96.95 & 88.27 & 96.11 & 92.21 \\
\hline LocalObjectPattern [14] & 95.32 & 92.54 & 90.27 & 93.03 \\
\hline
\end{tabular}

the pixel-level. Thus, they are more susceptible to noise, which is typically observed at the pixel-level in histopathological images. As seen in Table I, this might be the main reason for these algorithms to give lower accuracies compared to the objectbased algorithms, which do not directly use pixels in defining their features.

Among the object-based comparison methods, the DelaunayTriangulation algorithm, which represents an image with the distribution of only the nucleus components, yields the lowest accuracies. This is attributed to the importance of defining multityped objects for image representation. As aforementioned, the other object-based comparison algorithms also use multityped objects, which partially or entirely correspond to histological tissue components. However, to define these objects, they quantize image pixels into three clusters based on color information and then locate circles on each of these clusters. Different than these methods, our proposed model defines its objects, which may correspond to individual histological components but also local subregions of different characteristics, by using texture information in its first tier and scale (size and shape) information in its second tier. The comparison results indicate the effectiveness of this new object definition.

\section{A. Discussions}

In this paper, we conduct additional experiments in order to understand the effectiveness of the proposed two-tier tissue decomposition model. To this end, we make modifications in the model's steps and analyze the effects of these modifications on the system performance. First, we investigate the effectiveness of using an object-based representation instead of directly using the pixels' values. For that, we define two more representations that employ the filter outputs and the pixel clusters that we found in the first tier of our model. Particularly, the SchmidFilterBank algorithm represents an image with the outputs' averages and standard deviations separately calculated for each of the 13 filters and the QuantizedPixels algorithm extracts a bag-of-words representation on the pixel clusters. We give the comparison results obtained on the test set in Table II. As seen in this table, defining objects in the first tier greatly improves the classification results.

We then analyze the effectiveness of the second tier of our proposed model. For that, we remove this tier from the model and just use the characterized objects defined at the end of the
TABLE II

Test Set Results Obtained By the Modified Versions of the Proposed Two-TIER TISSUE DECOMPOSITION MODEL

\begin{tabular}{|l|c|c|c|c|}
\hline & Normal & Low & High & Overall \\
\hline Two-tier model & 99.18 & 93.83 & 93.77 & 95.47 \\
\hline Modifications & & & & \\
\hline SchmidFilterBank & 87.57 & 76.06 & 86.77 & 81.34 \\
\hline QuantizedPixels & 91.85 & 86.02 & 87.94 & 88.13 \\
\hline OnlyFirstTier & 97.96 & 89.57 & 92.60 & 92.65 \\
\hline OnlySecondTier & 77.59 & 66.94 & 90.66 & 74.05 \\
\hline AreaCircularityMetric & 99.19 & 89.81 & 93.77 & 93.34 \\
\hline TwoTierModelWithColor & 90.02 & 80.33 & 87.16 & 84.42 \\
\hline OnlyFirstTierWithColor & 98.98 & 85.07 & 78.60 & 86.24 \\
\hline
\end{tabular}

first tier. Thus, the OnlyFirstTier algorithm defines a graph on the objects and labels the graph edges with respect to the object types $\alpha_{i}$ defined by the first tier. The results given in Table II show that the second tier, which further categorizes the objects using the proposed $\mathrm{db}$ s metric, is useful in obtaining higher accuracies especially for images of low-grade cancerous tissues. Then, we make such kind of analysis for the first tier. However, since objects are defined using the clusters found by the first tier, we can only remove the object characterization part of this tier. In other words, in the OnlySecondTier algorithm, we still continue using the objects defined by the first tier but categorize these objects with respect to only their discretized dbs values $\beta_{i}$. The results reveal that the characterization of the objects based on texture information is necessary for accurate classifications.

Additionally, in the second tier, we use another set of metrics to further categorize the objects instead of using our proposed dbs metric. In the AreaCircularityMetric algorithm, the first tier identifies the precategories $\alpha_{i}$ of objects and then the second tier uses the objects' areas and circularities for their subcategorization (i.e., this method also uses $\alpha_{i}$ defined by the first tier but employs the objects' areas and circularities instead of their $\mathrm{db}$ s values). Here we define the circularity measure of an object as $4 \pi A / P^{2}$, where $A$ and $P$ are the area and perimeter of the object. The classification accuracies given in Table II show that the dbs metric is more effective to quantify the object's shape and size.

We also examine the effects of using texture to quantize image pixels. In the TwoTierModelWithColor algorithm, we cluster the pixels according to their RGB values, instead of the outputs of the Schmid's filters, and leave the rest of the proposed method the same. In the OnlyFirstTierWithColor algorithm, we also cluster the pixels based on their RGB values but also remove the second tier from the method. The results given in Table II reveal that the accuracies are much lower than those obtained when the filter outputs are used in clustering. We attribute this to the following: better individual histological tissue components, which are usually homogeneous in terms of color, might be located when the RGB values are used. However, relying on color might decrease the performance for the localization of local subregions, which are typically heterogeneous in terms of color.

Our proposed model uses an approximate representation, in which the objects approximately correspond to histological tissue components and local tissue subregions. Hence, it may 


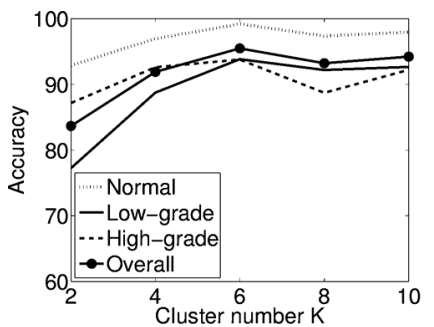

(a)

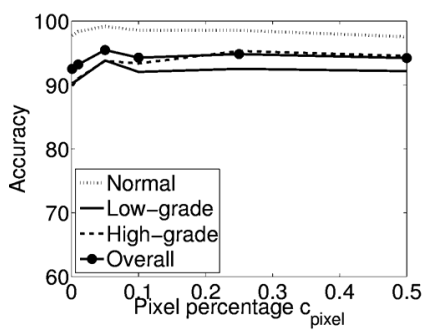

(c)

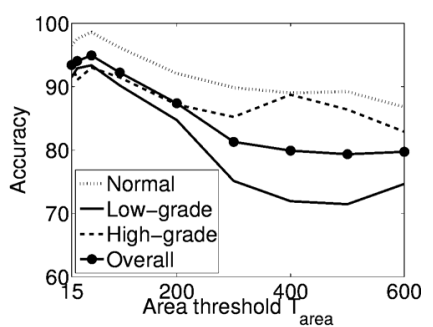

(b)

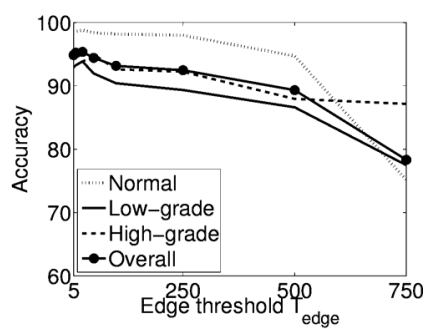

(d)
Fig. 9. Test set accuracies as a function of the model parameters: (a) cluster number $K$, (b) area threshold $T_{\text {area }}$, (c) pixel percentage $c_{\text {pixel }}$, and (d) edge threshold $T_{\text {edge }}$.

lead to noisy object definitions. To understand the effects of such objects, we conduct the following experiment. After automatically defining and labeling the objects, we visually examine some sample images and identify the object types that may correspond to the noisy objects. We then eliminate the objects of these identified types and run our method on the remaining objects. This experiment gives $95.16 \%$ overall test set accuracy $(98.98,93.00$, and $94.94 \%$ for the normal, low-grade cancerous, and high-grade cancerous classes, respectively). These accuracies are quite similar to those obtained when we use all object types. This experiment shows that refinements might be considered in object definition, which could be considered as a future work. However, such refinements necessitate human intervention, which would require a human expert in the system design.

\section{B. Parameter Analysis}

We also analyze the effects of parameter selection on the performance of the proposed model. To this end, for each parameter, we fix the other parameters and measure the test set accuracies when different values of this parameter are used. We present the test set accuracies as a function of each parameter in Fig. 9.

The first parameter is the cluster number $K$ to which image pixels are quantized in the first tier. In our model, we find connected components on the pixels of each cluster to define objects and use objects' clusters to find their precategories. Thus, this parameter determines the objects used in representation as well as their types. Selecting smaller $K$ values may result in defining single objects on the regions of different characteristics, decreasing classification accuracies. On the other hand, selecting larger values increases the number of the object types, which in turn increases the number of the edge types whose histogram will be used in feature extraction. As seen in Fig. 9(a), this slightly lowers the accuracies most probably due to curse-ofdimensionality. The other parameter used by the first tier is the area threshold $T_{\text {area }}$ to eliminate smaller connected components. Smaller threshold values cause to include spurious noisy objects into representation whereas its larger values cause to eliminate some necessary objects. Both of these conditions decrease the accuracy, as observed in Fig. 9(b).

The next parameter is the percentage $c_{\text {pixel }}$ used in dbs calculation in the second tier. When it is selected too small, objects are covered by a filter in the very first iterations, regardless of their shapes and scales, and hence the calculated $\mathrm{dbs}$ values cannot differentiate the objects. In that case, the model converges to the OnlyFirstTier algorithm, which we implemented for comparison purpose by removing the second tier from the model. Consistent with the comparison results provided in Table II, such selection lowers the classification accuracy. Selecting a larger value for this percentage slightly affects the results, as given in Fig. 9(c). However, as this parameter affects the point where the iterations stop, larger values will make the dbs calculation unnecessarily long.

The edge threshold $T_{\text {edge }}$ is the last parameter that the image representation/classification step uses to eliminate edge types with lower frequencies. Selecting too small values leads to using too much features in representation. This slightly reduces the accuracy, which is also attributed to curse-of-dimensionality in classification. When it is selected too large, only few features are left in representation and these features are not sufficient to accurately classify the images. The analysis of this parameter is given in Fig. 9(d).

\section{CONCLUSION}

This paper presents a new two-tier tissue decomposition model that defines multityped objects for histopathological image representation and uses the distribution of these objects for its classification. In the first tier of this model, it proposes to decompose an image into objects and precategorize these objects based on texture information. Then, in its second tier, it introduces the dominant blob scale metric to quantify the size and shape of an object with a single scalar and proposes to use this scalar for further categorization of the objects. At the end, it constructs a graph on the categorized objects and uses the histogram of graph edges, which are labeled according to the types of their end objects, for histopathological image classification. We tested our method on 3236 microscopic histopathological images of colon tissues. Our experiments revealed that the categorized objects, which are defined using the proposed two-tier tissue decomposition model, provide distinctive representations for normal and cancerous images, which lead to more accurate classification results compared to the existing algorithms.

One future research direction is to use the objects, defined and categorized by the proposed decomposition model, for image segmentation. For example, one may implement a region growing algorithm on the objects, in which the growing process is guided by the edge similarities of adjacent objects. This work constructs a graph to quantify the distribution of the categorized objects. However, one may investigate the other ways of this quantification, such as defining texture measures on these objects. Although we conducted our experiments on colon tissues, this method could also be used for different tissue 
types. In this case, the method should be trained to learn the object types specific to a tissue type. This would be another future research direction of the paper.

\section{REFERENCES}

[1] A. Tabesh, M. Teverovskiy, H. Y. Pang, V. P. Kumar, D. Verbel, A. Kotsianti, and O. Saidi, "Multifeature prostate cancer diagnosis and Gleason grading of histological images," IEEE Trans. Med. Imag., vol. 26, no. 10, pp. 1366-1378, Oct. 2007.

[2] F. Bunyak, A. Hafiane, and K. Palaniappan, "Histopathology tissue segmentation by combining fuzzy clustering with multiphase vector level sets," Adv. Exp. Med. Biol., vol. 696, pp. 413-424, 2011.

[3] A. N. Esgiar, R. N. G. Naguib, B. S. Sharif, M. K. Bennett, and A. Murray, "Microscopic image analysis for quantitative measurement and feature identification of normal and cancerous colonic mucosa," IEEE T. Inf. Technol. Biomed., vol. 2, no. 3, pp. 197-203, Sept. 1998.

[4] S. Doyle, M. Feldman, J. Tomaszewski, and A. Madabhushi, "A boosted Bayesian multiresolution classifier for prostate cancer detection from digitized needle biopsies," IEEE Trans. Biomed. Eng., vol. 59, no. 5, pp. 1205-1218, May 2012.

[5] K. Jafari-Khouzani and H. Soltanian-Zadeh, "Multiwavelet grading of pathological images of prostate," IEEE Trans. Biomed. Eng., vol. 50, no. 6, pp. 697-704, Jun. 2003.

[6] S. Doyle, S. Agner, A. Madabhushi, M. Feldman, and J. Tomaszewski, "Automated grading of breast cancer histopathology using spectral clustering with textural and architectural image features," in Proc. Biomed Imag.: From Nano to Macro, May 2008, pp. 496-499.

[7] O. Sertel, J. Kong, H. Shimada, U. V. Catalyurek, J. H. Saltz, and M. N. Gurcan, "Computer-aided prognosis of neuroblastoma on whole slide images: Classification of stromal development," Pattern Recogn., vol. 42, no. 6, pp. 1093-1103, Jun. 2009.

[8] Y. Zhang, B. Zhang, F. Coenen, and W. Lu, "Breast cancer diagnosis from biopsy images with highly reliable random subspace classifier ensembles," Mach. Vis. Appl., vol. 24, no. 7, pp. 1405-1420, Oct. 2013.

[9] A. N. Basavanhally, S. Ganesan, S. Agner, J. P. Monaco, M. D. Feldman, J. E. Tomaszewski, G. Bhanot, and A. Madabhushi, "Computerized image-based detection and grading of lymphocytic infiltration in HER2+ breast cancer histopathology," IEEE Trans. Biomed. Eng., vol. 57, no. 3, pp. 642-653, Mar. 2010.
[10] C. Demir, S. H. Gultekin, and B. Yener, "Learning the topological properties of brain tumors," IEEE ACM T. Comput. Bi., vol. 2, no. 3, pp. 262-270, Jul. 2005.

[11] B. Weyn, G. van de Wouwer, S. Kumar-Singh, A. Van Daele, P. Scheunders, E. van Marck, and W. Jacob, "Computer-assisted differential diagnosis of malignant mesothelioma based on syntactic structure analysis," Cytometry, vol. 35, pp. 23-29, Jan. 1999.

[12] D. Altunbay, C. Cigir, C. Sokmensuer, and C. Gunduz-Demir, "Color graphs for automated cancer diagnosis and grading," IEEE Trans. Biomed. Eng., vol. 57, no. 3, pp. 665-674, Mar. 2010.

[13] E. Ozdemir and C. Gunduz-Demir, "A hybrid classification model for digital pathology using structural and statistical pattern recognition," IEEE Trans. Med. Imag., vol. 32, no. 2, pp. 474-483, Feb. 2013.

[14] G. Olgun, C. Sokmensuer, and C. Gunduz-Demir, "Local object patterns for tissue image representation and cancer classification," IEEE J. Biomed. Health Inf., 2014, to be published.

[15] T. Lindeberg, "Feature detection with automatic scale selection," Int. J. Comput. Vis., vol. 30, no. 2, pp. 79-116, Nov. 1998.

[16] D. G. Lowe, "Distinctive image features from scale invariant keypoints," Int. J. Comput. Vis., vol. 60, no. 2, pp. 91-110, Nov. 2004.

[17] K. S. Pedersen, M. Loog, and P. van Dorst, "Salient point and scale detection by minimum likelihood," J. Mach. Learn. Res. Proc. Track, pp. 59-72, Jan. 2007.

[18] M. Isard and J. MacCormick, "BraMBLe: A Bayesian multiple-blob tracker," in Proc. IEEE Int. Conf. Comp. Vision, Jul. 2001, vol. 2, pp. $34-41$.

[19] R. T. Collins, "Mean-shift blob tracking through scale space," in Proc. IEEE Int. Conf. Comp. Vision and Pattern Recogn., Jun. 2003, vol. 2, pp. 234-240.

[20] P. Forssen and A. Moe, "View matching with blob features," in Proc. Canadian Conf. Comp. Robot Vision, May 2005, pp. 228-235.

[21] J. Yang, J. Cheng, and H. Lu, "Human activity recognition based on the blob features," in Proc. IEEE Int. Conf. Multimedia and Expo., Jun. 2009, pp. 358-361.

[22] Q. Xu and Y. Q. Cheng, "Multiscale blob features for gray scale, rotation and spatial scale invariant texture classification," in Proc. Int. Conf. Pattern Recogn., Aug. 2006, pp. 29-32.

[23] C. Schmid, "Constructing models for content-based image retrieval," in Proc. IEEE Int. Conf. Comp. Vis. Pattern Recog., Jun. 2001, vol. 2, pp. 39-45. 